Lepr Rev (2000) 71, 344-354

\title{
The hand-foot impairment score as a tool for evaluating prevention of disability activities in leprosy: an exploration in patients treated with corticosteroids
}

\author{
S.M. BROEKHUIS ${ }^{+}$, A. MEIMA ${ }^{+}$, L.F. KOELEWIJN ${ }^{+}$, \\ J.H. RICHARDUS ${ }^{+}$, C. BENBOW* \& \\ P.R. SAUNDERSON* \\ ${ }^{+}$Department of Public Health, Erasmus University Rotterdam, The \\ Netherlands \\ *All Africa Leprosy, Tuberculosis and Rehabilitation Training \\ Centre, Addis Ababa, Ethiopia
}

Accepted for publication 1 July 2000

\begin{abstract}
Summary The hand-foot (HF) impairment score in leprosy patients is the sum of the WHO disability grades for hands and feet. This retrospective study explored the possibility of using the HF score for evaluation of the effectiveness of corticosteroid treatment programmes for nerve function impairment (NFI). Changes in the score were compared with changes in sensory testing (ST) and voluntary muscle testing (VMT) for 42 leprosy patients who received corticosteroid treatment. The WHO grade did not change in 30/60 (50\%) of extremities gaining, and in 4/10 (40\%) extremities losing sensation and/or muscle strength. However, 18/24 (75\%) patients with a definite gain in function improved in HF score, while the HF score remained unchanged in 10/11 (91\%) patients with no change in nerve function. Five patients with impairment in multiple extremities showed both gain and loss of sensation and/ or muscle strength in the same or different extremities. Overall, improvement, deterioration and absence of change in NFI, as indicated by changes in ST and VMT were reflected correctly by the HF score in $28(76 \%)$ of the remaining 37 patients. It was also shown that the HF score does not give appropriate information on the extent of the effect of corticosteroid treatment. This study illustrates that the HF score can not be used to support management of corticosteroid treatment of individual patients, but indicates this score to be a promising device for the evaluation of the effectiveness of corticosteroid treatment programmes. This study used the HF score because information on (changes in) eye impairment was not considered reliable. However, in principle, we consider the EHF score, which is the sum of the WHO disability grades for hands, feet and eyes, preferable for evaluation purposes. We strongly recommend further validation of the EHF score as a tool for evaluation of corticosteroid treatment programmes for patient groups with different distributions of NFI through prospective studies.
\end{abstract}

Correspondence to: P.R. Saunderson, ALM, 1 ALM Way, Greenville SC 29601, USA (e-mail: psaunderson@ leprosy.org) 


\section{Introduction}

In view of the forecast elimination of leprosy as a public health problem, changes in leprosy control programmes are inevitable. The diminishing number of patients on multidrug treatment (MDT) in particular leads to more emphasis on the prevention of impairments and disabilities (POID). POID projects have now been implemented in many leprosy control programmes.

One important activity to prevent development of disabilities is to treat patients who suffer from recent nerve function impairment (NFI) with corticosteroids. Registration of nerve function assessments is required in individual patients treated this way. Ideally, POID programmes provide patient cards which include information on these assessments. The interpretation of subsequent assessments is of ten complicated because several nerves and many testing sites are involved. Adequate methods for interpreting nerve function assessments could lead to better management of individual patients treated with corticosteroids.

In addition, there is a need to evaluate the effectiveness of POID activities, such as treatment with corticosteroids, at programme level. This calls for methods of scoring impairment and disability that are sufficiently accurate to determine the relevant changes induced by POID activities for the related groups of patients. A further requirement is that it should be feasible to collect and process the field data from which the scores are to be derived. Evaluation at programme level poses less strict demands on the sensitivity of scoring methods than are required for individual patient management.

In the field, the standard method of detecting recent NFI is to carry out sensory testing (ST) and voluntary muscle testing (VMT) on a regular basis. The decision of the health worker to treat the patient with corticosteroids is based on the patient's history as determined at intake, and on initial ST and VMT results and subsequent changes. One method used to track changes in sensation and muscle strength between start and end of multidrug treatment for groups of patients is to combine point-wise data on ST and VMT results for individual nerves. ${ }^{1,2}$ However, it is not considered feasible to computerize data on ST and VMT for all points and nerves tested on a routine basis in a field programme.

At present, the main indicator used to assess impairments and disabilities is the WHO disability grading system. This score has three possible outcomes $(0,1$ and 2$)$ that are determined by different types of information: sensory testing results and the presence of visible deformity or damage. Eyes, hands and feet of the patient are given a score, and the part of the body with most damage determines the overall score of the patient. ${ }^{3}$ This 'maximum WHO disability grade' is at present primarily used for statistical purposes to compare the impairment and disability status of newly detected patients across countries. ${ }^{4}$ It is, however, questionable whether the crude WHO disability grading system is an appropriate tool to evaluate the effectiveness of POID activities.

In 1994, de Rijk et al. ${ }^{5}$ introduced two scores which are related to the WHO disability grading system: the hand-foot score (HF) score and the eye-hand-foot (EHF) score. These scores describe the severity of the patient's disability in more detail, because they are the summation of the WHO disability grades for the extremities (HF score: maximum value 8) and for the extremities and eyes (EHF score: maximum value 12). The HF score can be used instead of the EHF score when the reliability of data on eye impairment is limited.

Validation of impairment and disability scoring methods is necessary for sensible application in leprosy control. This paper explores the validity of the WHO disability grading system and the HF score in the context of one specific POID activity: corticosteroid 
Table 1. WHO disability grades for hands and feet as defined in 1988 by the WHO Expert Committee on Leprosy

\begin{tabular}{ll} 
Grade & Condition of the hand or foot \\
\hline 0 & No anaesthesia, no visible deformity or damage due to leprosy \\
1 & Anaesthesia present, no visible deformity or damage due to leprosy \\
2 & Visible deformity or damage present due to leprosy \\
\hline
\end{tabular}

treatment. Two issues are addressed, namely their potential (1) to assess impairment changes relevant to the individual patient in order to support patient management, and (2) to serve as tools for evaluation of the effectiveness of treatment with corticosteroids as a programme activity. This is done by investigating how well changes in the WHO disability grades for extremities, the maximum WHO disability grade and the HF score relate to changes in ST and VMT results in extremities and patients before and after corticosteroid treatment.

\section{Materials and methods}

This study was conducted at the All African Leprosy, Tuberculosis and Rehabilitation Training Centre (ALERT) in Addis Ababa, Ethiopia. Included in this study are a selection of patients who were treated with corticosteroids in the past 8 years because of recent or threatening NFI. The study reports retrospectively on the findings of nerve function assessments in these patients as performed in ALERT's field programme. This group of patients was not part of the ALERT MDT Field Evaluation Study (AMFES), although they were managed in the same programme and according to the same guidelines as patients in that study. These patients were selected on the basis of having had a course of steroids in the previous three years and their prior history of nerve damage was variable.

ALERT field workers carry out ST and VMT on a regular basis for each patient in order to detect NFI. Sensory testing is conducted by applying a $10 \mathrm{~g}$ filament to 10 points on the palm and fingers of the hand, and to 10 points on the sole and toes of the foot. Points for which the $10 \mathrm{~g}$ filament is not felt are marked on the patient card. For this study, the possible ST outcomes 'felt' and 'not felt' were assigned 0 and 1 point, respectively. Voluntary muscle testing is done for eight nerves: left and right facial nerves, left and right ulnar nerves, left and right median nerves and left and right peroneal nerves. The results of these tests are documented as 'strong', 'weak' or 'paralysed' on the ALERT field programme patient card.

ST and VMT are also conducted at the start and end of treatment with corticosteroids. On both occasions, the patients' ST results (40 points) and VMT results (eight nerves) are recorded on the ALERT prednisolone treatment and release form. WHO disability grades for the extremities and eyes at the start and end of treatment are also specified on this form. At the time of this study, the 1988 WHO disability grading system was used (see Table 1). This paper analyses to what extent changes in ST and VMT results are reflected in changes in WHO disability grades for individual extremities (extremity level), and to what extent overall changes in ST and VMT results for patients are reflected in changes in the maximum WHO disability grade and HF score (patient level). Reporting on impairment changes of eyes was not considered reliable enough for this study. Eye impairments are therefore excluded from 
the present analysis and disability grades for the eyes were not considered in the calculation of the maximum WHO disability grades. Similarly, the HF score was used instead of the EHF score.

In order to investigate to what extent changes in ST and VMT results are reflected in changes in WHO disability grades at extremity level, changes in ST and VMT of the extremities were categorized as follows:

- 'Full recovery': an improvement in ST of 2 points or more and/or an improvement in VMT, with complete absence of NFI at the end of treatment.

- 'Recovery': an improvement in ST of 2 points or more, without sensory loss at the end of treatment, but with some remaining loss of muscle strength, or vice versa (an improvement in VMT with normal muscle strength at the end of treatment, but with some remaining sensory loss).

- 'Improvement': an improvement in ST of 2 points or more and/or an improvement in VMT, in hands, for either the ulnar or median nerve or both, with some NFI remaining at the end of treatment.

- 'No change': no change in ST and VMT, or an improvement or deterioration in ST of 1 point and no change in VMT.

- 'Deterioration': deterioration in ST of 2 points or more and/or deterioration in VMT.

- 'Both': improvement in ST of 2 points or more and deterioration in VMT, or vice versa.

In this definition, 'recovery' in a hand through regained motor function implies that the VMT result is 'strong' for both the median and the ulnar nerve. Improvement (and the converse, deterioration) in ST by 2 points or more is defined so as not to occur together with deterioration (and the converse, improvement) in the categories 'full recovery', 'recovery', 'improvement' and 'deterioration'.

In order to investigate to what extent overall ST and VMT changes in patients are reflected in changes in the maximum WHO disability grades and HF scores, changes in ST and VMT have also been categorized at patient level. This was, on the basis of the categorization for extremities, done as follows:

- 'Full recovery': full recovery of both sensation and muscle strength in at least one extremity, without 'deterioration' in other extremities.

- 'Recovery': full recovery of sensation or full recovery of muscle strength in at least one extremity, without 'deterioration' in that and other extremities, but with (some) remaining NFI in that extremity at the end of treatment.

- 'Improvement': improvement in sensation and/or muscle strength in at least one extremity, without 'deterioration' in that and other extremities, also with (some) NFI remaining in that extremity at the end of treatment.

- 'No change': no change in any extremity.

- 'Deterioration': deterioration in sensation and/or muscle strength in at least one extremity, without improvement in that and other extremities.

- 'Both': improvement or full recovery in at least one extremity and deterioration in the same or another extremity.

For example, a patient with an improvement in sensation in the right hand and a recovery of muscle strength, with no change in the left hand, and with improvements in sensation of the feet is recorded as a 'recovery'. 
Table 2. Distribution of the maximum WHO disability grade and HF score among the 42 study patients at the start of treatment with corticosteroids

\begin{tabular}{lrr}
\hline Maximum & & \\
WHO grade & Number & Percentage \\
\hline 0 & 4 & $10 \%$ \\
2 & 14 & $33 \%$ \\
Total & 42 & $100 \%$ \\
\hline HF score & Number & Percentage \\
\hline 0 & 4 & $10 \%$ \\
1 & 2 & $5 \%$ \\
2 & 11 & $26 \%$ \\
3 & 7 & $17 \%$ \\
4 & 13 & $31 \%$ \\
5 & 1 & $2 \%$ \\
6 & 3 & $7 \%$ \\
7 & 0 & $0 \%$ \\
8 & 1 & $2 \%$ \\
Total & 42 & $100 \%$ \\
& & \\
\hline
\end{tabular}

\section{Results}

A total of 42 patients were included in this analysis. Thirty-three (78.6\%) of the patients were classified as multibacillary (MB) and nine $(21.4 \%)$ as paucibacillary (PB). The mean age was 33.4 years (range 16-56). The patients suffered from different types of reactions requiring different steroid regimes. The time between the assessment at the start of the treatment and the assessment at the time that it was decided to stop treatment varied considerably: from less than 3 months (four patients) to more than 1 year (again four patients). At the start of the corticosteroid treatment, three out of the $42(7 \%)$ study patients had no extremity affected with NFI as diagnosed through ST and VMT. Again 3/42 (7\%) had one extremity affected, $11 / 42$ (26\%) had two, 11/42 (26\%) had three and 14/42 (33\%) had four. NFI was present in 114 out of the $168(4 \times 42,68 \%)$ involved. The distributions of the maximum WHO disability grade and the HF score of the patients at the start of treatment are shown in Table 2.

\section{CHANGES IN EXTREMITIES}

Table 3 shows the changes in ST and VMT in the 168 extremities of the 42 patients, and the relation between those changes. Out of the 114 extremities with NFI at the start of corticosteroid treatment, $60(53+7=60,53 \%$, see Table 3$)$ had partial or complete return of nerve function. Improvement of sensation only, was observed in $36(60 \%)$ of these 60 extremities. This figure is $7 / 60(12 \%)$ for partial or complete return of motor function only. One extremity lost sensation and gained motor function.

Table 4 compares the changes in ST and VMT with the changes in the WHO grade for the individual extremities. 21/23 improvements in extremities are not rewarded with a change in WHO disability grades. Also, 7/12 extremities with a recovery, and 2/25 extremities with a 
Table 3. Changes in ST and VMT between start and end of corticosteroid treatment in individual extremities*

\begin{tabular}{|c|c|c|c|c|}
\hline Change in VMT & $\begin{array}{c}\text { Change in ST } \\
\text { Improvement } \\
\text { or recovery }\end{array}$ & No change & Deterioration & Total \\
\hline Improvement or recovery & 17 & 7 & 0 & 24 \\
\hline No change & 36 & 98 & 10 & 144 \\
\hline Deterioration & 0 & 0 & 0 & 0 \\
\hline Total & 53 & 105 & 10 & 168 \\
\hline
\end{tabular}

* At the start of corticosteroid treatment, 59/105 extremities with no change in ST had full sensation, 123/144 extremities with no change in VMT had normal muscle strength, and 48/98 extremities with neither a change in ST nor in VMT had both full sensation and normal muscle strength.

full recovery do not improve in WHO grade. This implies that 50\% (30/60) of the improvements, recoveries and full recoveries in extremities are not reflected in an improvement in the WHO grading system. Out of these 30 extremities, 27 were not free from sensory loss at the end of corticosteroid treatment. Sensation returned in 3.4 points on average (range: 1 point deterioration to 7 points improvement) with 24 extremities at least showing improvement. On further analysis of these 30 extremities, recovery in motor function was seen in eight of the 17 extremities with initial loss of motor function. The WHO grading system also did not pick up 4/10 (40\%) deteriorating extremities. In all, 90/98 (92\%) of the extremities with no relevant change (1 point ST or less and no change in VMT) in nerve function showed no change in WHO grade.

The WHO grade improved in 30/60 (50\%) of the extremities with some gain in nerve function. This was seen in $2 / 23(9 \%)$ extremities with improvement, $5 / 12(42 \%)$ extremities with a recovery, and in $23 / 25(92 \%)$ extremities with a full recovery. Twenty-eight of these 30 extremities recovered from sensory loss, one remained with sensory loss (the WHO grade changed from 2 to 1 ), and the average number of points gaining back sensation was 5.5 points (range of improvement: $0-10$ points). The motor function recovered in six out of the 11 extremities with initial loss of motor function amongst these 30. In only two of these 30 extremities did the WHO grade improve from 2 to 0 . The WHO grade deteriorated by 1 point in $6 / 10(60 \%)$ extremities that deteriorated.

Table 4. Comparison of changes in ST and VMT and changes in WHO disability grades between start and end of corticosteroid treatment for the individual extremities*

\begin{tabular}{lcccrrr}
$\begin{array}{l}\text { Change in WHO } \\
\text { grade }\end{array}$ & Full recovery & Recovery & $\begin{array}{l}\text { Change in nerve functioning } \\
\text { Improvement }\end{array}$ & No change & Deterioration & Total \\
\hline-1 & 0 & 0 & 0 & 3 & 6 & 9 \\
0 & 2 & 7 & 21 & 90 & 4 & 124 \\
1 & 23 & 4 & 1 & 4 & 0 & 32 \\
2 & 0 & 1 & 1 & 98 & 10 & 168 \\
Total* & 25 & 12 & 23 & & & \\
\end{tabular}

\footnotetext{
* No extremities showed improvement in ST and deterioration in VMT or vice versa (category 'Both') . At the start of corticosteroid treatment, 48/98 extremities with neither a change in ST nor in VMT had both full sensation and normal muscle strength, and 55/124 extremities with no change in WHO grade had grade 0 .
} 
Table 5. Comparison of overall changes in nerve functioning and changes in maximum WHO disability grade between start and end of corticosteroid treatment for the study patients.

\begin{tabular}{|c|c|c|c|c|c|c|c|}
\hline \multirow{2}{*}{$\begin{array}{l}\text { Change } \\
\text { in maximum } \\
\text { WHO grade }\end{array}$} & \multirow[b]{2}{*}{ Full recovery } & \multirow[b]{2}{*}{ Recovery } & \multicolumn{3}{|c|}{ Overall change in nerve functioning } & \multirow[b]{2}{*}{ Both } & \multirow[b]{2}{*}{ Total } \\
\hline & & & Improvement & No change & Deterioration & & \\
\hline-1 & 1 & 0 & 0 & 1 & 0 & 0 & 2 \\
\hline 0 & 11 & 4 & 3 & 10 & 2 & 5 & 35 \\
\hline 1 & 5 & 0 & 0 & 0 & 0 & 0 & 5 \\
\hline Total* & 17 & 4 & 3 & 11 & 2 & 5 & 42 \\
\hline
\end{tabular}

* Changes in maximum WHO grade equal to -2 or +2 did not occur.

\section{CHANGES IN PATIENTS}

Changes in nerve function of patients are compared with the change in the maximum WHO disability grade in Table 5. It is seen that gain in nerve function is missed by the maximum WHO grade in 19/24 (79\%) patients, including $12 / 17$ (71\%) patients with a full recovery in at least one extremity.

The results of the comparison between changes in nerve function and changes in the HF score are shown in Table 6 . In the 42 study patients, changes in the HF score were as follows: improvement 19/42 (45\%), no change 19/42 (45\%) and deterioration 4/42 (10\%). Overall changes in nerve function were as follows: any gain 24/42 (57\%), no change $11 / 42(26 \%)$, deterioration 2/42 (5\%), and both improvement and deterioration in ST and/or VMT in the extremities of the same patient 5/42 (12\%). The last group of patients (both improvement and deterioration in the same patient) is excluded from the discussion of Table 6 , below.

In $6 / 24(25 \%)$ patients with a gain in nerve function (i.e. improvement, recovery or full recovery), the HF score did not change. Out of these six, three patients had a ST improvement with some remaining loss of sensation in one extremity without other changes in sensation. This was accompanied by an improvement or recovery in motor function in the same extremity in two of them. In the third patient, motor function was always normal. The fourth patient had a total recovery in motor function in three extremities without changes in sensation. In the fifth patient sensation improved, but loss of sensation remained in two

Table 6. Comparison of overall changes in nerve functioning and changes in the HF score between start and end of corticosteroid treatment for the study patients

\begin{tabular}{|c|c|c|c|c|c|c|c|}
\hline $\begin{array}{l}\text { Change } \\
\text { in HF score }\end{array}$ & Full recovery & Recovery & \multicolumn{3}{|c|}{ Overall change in nerve functioning } & Both & Total \\
\hline-2 & 0 & 0 & 0 & 0 & 0 & 2 & 2 \\
\hline-1 & 0 & 0 & 0 & 1 & 0 & 1 & 2 \\
\hline 0 & 2 & 2 & 2 & 10 & 2 & 1 & 19 \\
\hline 1 & 10 & 0 & 1 & 0 & 0 & 0 & 11 \\
\hline 2 & 2 & 0 & 0 & 0 & 0 & 1 & 3 \\
\hline 3 & 1 & 1 & 0 & 0 & 0 & 0 & 2 \\
\hline 4 & 2 & 1 & 0 & 0 & 0 & 0 & 3 \\
\hline Total & 17 & 4 & 3 & 11 & 2 & 5 & 42 \\
\hline
\end{tabular}


extremities. In a third extremity, motor function improved but did not recover while all loss of sensation disappeared. The sixth patient recovered from loss of sensation in two extremities with recovery of motor function in one of them, but kept sensory loss and developed a visible deformity in the other two extremities.

The HF score did not register change in two patients with a deteriorating nerve function in at least one extremity. The HF score also remained the same in 10/11 (91\%) patients without changes in nerve function.

In $1 / 3$ patients with improvement and in $2 / 4$ patients with a recovery of either sensation or motor function in at least one extremity, the HF score improved. This is also seen in $15 / 17$ $(88 \%)$ patients with full recovery of both sensation and motor function in at least one extremity. The improvement in HF score ranged from 1 to 4 points in the $18(1+2+15=18)$ patients gaining both in nerve function and in HF score.

Overall, gain, deterioration and absence of change in nerve function impairment (NFI) as indicated by changes in ST and VMT were reflected correctly by the HF score in 28 $(10+18=28,76 \%)$ of the 37 patients who did not show both improvement and deterioration in ST and/or VMT in the same or different extremities.

\section{Discussion}

Leprosy is a serious disease because of the impairments and disabilities that it may cause. Measurement of impairment and disability is therefore of vital importance for leprosy control. Two major purposes for measuring can be distinguished: (1) support of individual patient management, and (2) evaluation of leprosy control activities. To meet the first purpose, measurement tools are required that detect new NFI, and changes in existing impairment and disability, early enough to enable appropriate decision making on POID activities for individual patients. The second purpose involves issues such as comparison of impairment and disability in new patients between areas or countries and over time, comparison of impairment and disability at detection, at release from MDT and during post-treatment surveillance, and evaluation of the effectiveness of POID programmes.

Several scoring systems have been introduced in the past. ${ }^{2,3,5-15}$. Undoubtedly, the most frequently used scoring system is the WHO disability grading system with the grades 0,1 and 2. ${ }^{3,14}$ In its latest update in 1998 , the grades for the eyes were re-defined. ${ }^{14}$ Some of these systems, including the WHO grading system, have been used to determine changes in impairment and disability between start of MDT, release from treatment (RFT) and (sometimes) post-treatment surveillance. ${ }^{1,2,5,13}$ The EHF score was shown to be more sensitive in detecting change in impairment and disability than the maximum WHO disability grade. ${ }^{16}$ The same was shown for a newer scoring system, that specifies proportions of patients who worsen in ST, VMT, wound count or bone loss. ${ }^{2}$

Nevertheless, it still remains attractive to use the simple WHO grading system and the directly associated HF or EHF score for assessment of changes in order to support patient management and to evaluate POID activities. In this study, ST and VMT results (which can be regarded as the 'gold standard' for measuring NFI) were used to explore the validity of the WHO grading system and the HF score to assess changes in patients that are relevant for patient management, and at aggregated level in order to evaluate POID programmes, in particular corticosteroid treatment. 
Firstly, changes in nerve function in individual extremities were compared with changes in WHO disability grade. Half of the extremities with gain in nerve function and $40 \%$ of the extremities that deteriorated were not detected by the WHO grading system. Most extremities with a gain in nerve function that were not recognized by the WHO grading system had improvement in sensation without recovery. Recovery in motor function was also missed frequently by the WHO grading system. Changes in ST and VMT in individual extremities are thus very poorly described by the WHO disability grading system. This is not surprising considering the definition of the WHO disability grading system, which ignores differences in the extent of loss of sensation and only accounts for loss of motor function that is accompanied by visible deformity (e.g. clawing, drop foot).

Secondly, changes in overall nerve functioning were compared with changes in the maximum WHO grade and HF score. The maximum WHO grade did not improve in 19/24 (79\%) patients with an improvement, a recovery or a full recovery in overall nerve function (Table 5). This is also not surprising because the maximum WHO grade only improves when all initially impaired extremities (eyes were not considered in this study) improve in WHO grade. All 19 patients with gain in nerve function but not in WHO grade had some remaining loss of sensation in one or more extremities (data not shown in tables).

The HF score, which is the sum of the WHO grades of the individual extremities, is more sensitive to change. The proportion of patients for whom an improvement, a recovery or a full recovery in nerve function was rewarded with improvement in HF score was 18/24 (75\%). In addition, gain, deterioration and absence of change in nerve function was reflected correctly by the HF score in $28(76 \%)$ of the 37 patients who did not show both improvements and deteriorations in ST and/or VMT. These proportions are low from the perspective of individual patients, but the HF score does indicate change in overall nerve function in a definite majority of patients in the study group. Further analysis revealed that the HF score does not provide appropriate information about the degree of nerve function change: $7 / 11$ patients with a change of 1 point in the HF score gained in nerve function in at least two extremities.

Because their sensitivity in recognizing ST and VMT changes in the individual patient is clearly insufficient, the individual WHO disability grades, the maximum WHO disability grade and the HF score are not at all suitable for supporting the management of NFI in individual patients. The WHO disability grading system and (E)HF score were definitely not developed for this purpose. The findings were expected but quantitative evidence was lacking so far. Alternative methods to support patient management have been described elsewhere. ${ }^{15,16}$

The HF score appeared adequate for the study group as a whole. The small group size and retrospective nature are clear limitations of our explorative study. The study group is a random mix of cases that received treatment with corticosteroids, reflecting field programme conditions. Our results suggest that the HF score has the potential to give an overall picture of the effectiveness of corticosteroid treatment under such conditions. Our study group was affected considerably by NFI; at the start of corticosteroid treatment, $60 \%$ of patients had three or four extremities affected. One might expect that in a different case mix with a lower level of NFI, more patients would fully recover from nerve function loss when given corticosteroids. In such situations, the HF score may give an even better reflection of the success of treatment with corticosteroids. This assumption needs further substantiation.

In this study, we used the HF score because information on (changes in) eye impairment was not considered reliable. Compared with other scoring systems, the HF and EHF score 
have the advantage of being very simple and reproducible; they are based on information that is already routinely collected in many programmes (the WHO grades for extremities and eyes). For possible future evaluation, we advocate use of the EHF score instead of the HF score because eye impairment is very important, its prevalence varies greatly in different patient populations, and score results should be comparable between different projects and centres. $^{16}$

The limitations of our study necessitate further validation studies involving larger patient groups before the EHF score can be used effectively for evaluation of POID programmes. The extent to which changes in nerve function in patient groups with different mixtures of complications leading to NFI are reflected in changes in the EHF score should be investigated in prospective studies. When further validated in this way, the EHF score will be useful for evaluating the effectiveness of different corticosteroid treatment programmes. Another issue that needs clarification through such studies relates to the significance of what is, and what is not detected by the EHF score. To be an effective tool in programme management, the EHF score must be sensitive enough to detect those levels of change in nerve function that lead to lasting disabilities and handicap.

Other POID activities such as health education, provision of footwear and reconstructive surgery also call for evaluation. It is appreciated that different POID activities have different outcomes in terms of health, physical ability, and social functioning and acceptability. Different POID activities may thus require scoring methods other than the EHF score for effective evaluation.

\section{Acknowledgements}

We thank ALERT, Dr Assefa Amenu and the staff of the Leprosy Control Division for help in carrying out this study. ALERT is supported by ILEP members, co-ordinated by Netherlands Leprosy Relief.

\section{References}

${ }^{1}$ Richardus JH, Finlay KM, Croft RP, Smith WC. Nerve function impairment in leprosy at diagnosis and at completion of MDT: a retrospective cohort study of 786 patients in Bangladesh. Lepr Rev, 1996; 67: 297-305.

2 Schreuder PA. The occurrence of reactions and impairments in leprosy: experience in the leprosy control program of three provinces in Northeastern Thailand, 1978-1995. III. Neural and other impairments. Int J Lepr Other Mycobact Dis, 1998; 66: 170-181.

3 WHO Expert Committee on Leprosy. WHO Technical Report Series No. 786. World Health Organization, Geneva, 1988.

4 WHO. Leprosy disabilities: magnitude of the problem. Weekly Epidemiol Rec, 1995; 70: 269-275.

${ }^{5}$ de Rijk AJ, Gabre S, Byass P, Berhanu T. Field evaluation of WHO-MDT of fixed duration at ALERT, Ethiopia: the AMFES project - I. MDT course completion, case-holding and another score for disability grading. Le pr Rev, 1994; 65: 305-319.

6 WHO Expert Committee on Leprosy. WHO Technical Report Series No. 189. World Health Organization, Geneva, 1960.

7 WHO Expert Committee on Leprosy. WHO Technical Report Series No. 459. World Health Organization, Geneva, 1970.

8 Bechelli LM, Dominguez M. Disability index for leprosy patients. Bull World Health Org, 1971; 44: 709-713.

9 Hasan S. An appraisal of use of the classifications of disabilities resulting from leprosy in field work and suggestions for improvement. Lepr Ind, 1982; 54: 135-142.

10 Kulkami UP, Kulkarni VN, Jogaikar DG. Classification on disabilities as followed by Poona District Leprosy Committee. Ind J Lepr, 1984; 56: 269-279. 
11 Watson JM. WHO disability grading. Letter to the editor. Lepr Rev, 1985; 56: 172-175.

12 Brandsma JW, de Jong N, Tjepkema A. Disability grading in leprosy. Suggested modifications to the WHO disability grading form. Lepr Rev, 1986; 57: 361-369.

13 Ponnighaus IM, Boerrigter G, Fine PE, Ponnighaus JM, Russell J. Disabilities in leprosy patients ascertained in a total population survey in Karonga District, northern Malawi. Lepr Rev, 1990; 61: 366-374.

14 WHO Expert Committee on Leprosy. WHO Technical Report Series No. 874. World Health Organization, Geneva, 1998.

15 Anderson AM, Croft RP. Reliability of Semmes Weinstein monofilament and ballpoint sensory testing and voluntary muscle testing in Bangladesh. Lepr Rev, 1999; 70: 305-313.

16 van Brakel WH, Reed NK, Reed DS. Grading impairment in leprosy. Lepr Rev, 1999; 70: 180-188. 